\begin{tabular}{|c|c|c|c|}
\hline Asim Kichloo, MD & Michael M. Aljadah, MD & Farah Wani, MD & Sindhura Ananthaneni, MD \\
\hline $\begin{array}{l}\text { Department of Internal Medicine, } \\
\text { St. Mary's of Michigan, Saginaw, Ml; } \\
\text { Associate Professor of Internal Medicine, } \\
\text { Central Michigan University College of } \\
\text { Medicine, Saginaw, MI }\end{array}$ & $\begin{array}{l}\text { Department of Internal Medicine, } \\
\text { Medical College of Wisconsin Affiliated } \\
\text { Hospitals, Milwaukee, WI }\end{array}$ & $\begin{array}{l}\text { Department of Family Medicine, } \\
\text { Samaritan Medical Center, } \\
\text { Watertown, NY }\end{array}$ & $\begin{array}{l}\text { Department of Internal Medicine, } \\
\text { St. Mary's of Michigan, Saginaw, Ml; } \\
\text { Central Michigan University College of } \\
\text { Medicine, Saginaw, MI }\end{array}$ \\
\hline
\end{tabular}

\author{
Michael M. Aljadah, MD \\ Department of Internal Medicine, \\ Medical College of Wisconsin Affiliated
}

Medicine, Saginawi
Farah Wani, MD

ment of Family Medicine,

Watertown, NY
Sindhura Ananthaneni, MD
Department of Internal Medicine,
St. Mary's of Michigan, Saginaw, MI;
Central Michigan University College of

Medicine, Saginaw, MI

(1)

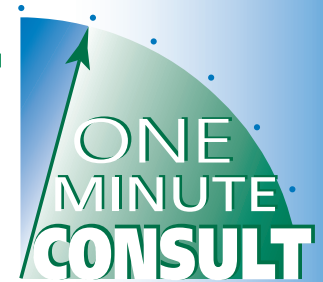

BRIEF ANSWERS

TO SPECIFIC

CLINICAL

QUESTIONS

\title{
Should we give triple therapy to patients with atrial fibrillation after percutaneous intervention?
}

\begin{abstract}
A:
No. In patients with atrial fibrillation undergoing percutaneous coronary intervention (PCI), the combination of an anticoagulant plus 2 antiplatelet drugs ("triple therapy") has been shown to lead to more bleeding events and a higher mortality rate than anticoagulation plus a single antiplatelet drug ("double therapy"). ${ }^{1}$

Patients should start double therapy immediately after PCI and continue for 1 year, before transitioning to a direct-acting oral anticoagulant (DOAC) by itself. ${ }^{1}$ If the patient is at high risk of a stroke but at low risk of bleeding, one can consider giving an anticoagulant plus 2 antiplatelet drugs (eg, clopidogrel plus aspirin) for a maximum of 1 month after PCI, and then stopping the aspirin for the remainder of the year. ${ }^{1}$ Patients should then be maintained on a DOAC by itself after 1 year, with risks and benefits considered.
\end{abstract}

\section{WHICH ANTICOAGULANT? WHICH ANTIPLATELET DRUGS?}

Until recently, there were no randomized controlled trials that measured the differences in bleeding events and death with anticoagulation plus 2 antiplatelet drugs compared with anticoagulation plus 1 antiplatelet drug. Clinically, triple therapy has generally meant an oral vitamin K antagonist (eg, warfarin) as the anticoagulant plus, for the antiplatelet drugs, a $\mathrm{P}_{2} \mathrm{Y}_{12}$ inhibitor (eg, clopidogrel) and aspirin. ${ }^{2}$ Double therapy has meant an oral anticoagulant plus a $\mathrm{P} 2 \mathrm{Y}_{12}$ inhibitor only. ${ }^{2}$

Of the P2 $\mathrm{Y}_{12}$ inhibitors, clopidogrel was found to pose the lowest risk of bleeding (fol- lowed by ticagrelor in selected patients) and should be the platelet inhibitor of choice. ${ }^{1}$ Of the oral anticoagulants, DOACs were found to be associated with fewer deaths and bleeding events than vitamin $\mathrm{K}$ antagonists and so should be the oral anticoagulants of choice. ${ }^{3,4}$ Thus, warfarin and other vitamin $\mathrm{K}$ antagonists are no longer recommended in the treatment of atrial fibrillation after PCI.

\section{BENEFITS OF ANTICOAGULATION}

From $20 \%$ to $30 \%$ of ischemic strokes in the United States are related to atrial fibrillation. ${ }^{5}$ Furthermore, nearly 3 million patients undergo PCI each year worldwide. ${ }^{5}$ Therefore, anticoagulation is imperative to reduce the risk of embolization secondary to cardiac blood stasis in patients with atrial fibrillation who are undergoing coronary PCI.

\section{EVIDENCE FOR DOUBLE THERAPY}

The WOEST trial (What Is the Optimal Antiplatelet and Anticoagulant Therapy in Patients With Oral Anticoagulation and Coronary Stenting?), ${ }^{2}$ in 2013, was the first randomized trial to address the optimal antiplatelet therapy in patients on oral anticoagulation undergoing PCI.

This open-label trial, specifically designed to detect bleeding events, found that oral anticoagulation plus clopidogrel (double therapy) caused less bleeding than anticoagulation plus clopidogrel plus aspirin (triple therapy). Furthermore, there was no excess of ischemic events or tradeoff in efficacy (eg, increased incidence of stroke, stent thrombosis, or myocardial infarction) with double therapy compared with triple therapy. ${ }^{2}$

\section{Triple therapy \\ is aggressive and carries higher risks of bleeding and death}


At 1 year, bleeding had occurred in 19.4\% of the double-therapy group and $44.4 \%$ of the triple-therapy group (hazard ratio [HR] 0.36, 95\% confidence interval [CI] 0.26-0.50, P < .0001 , number needed to treat 4 ). Also, $2.6 \%$ of the patients in the double-therapy group died, compared with $6.3 \%$ of the triple-therapy group (HR 0.39, 95\% CI 0.16-0.93, $P=$ .027 , number needed to treat 27). 2,6

The PIONEER AF-PCI trial (OpenLabel, Randomized, Controlled, Multicenter Study Exploring Two Treatment Strategies of Rivaroxaban and a Dose-Adjusted Oral Vitamin K Antagonist Treatment Strategy in Subjects With Atrial Fibrillation Who Undergo Percutaneous Coronary Intervention), ${ }^{3}$ in 2016, found that the DOAC rivaroxaban (in 2 different doses) in combination with a $\mathrm{P}_{2} \mathrm{Y}_{12}$ inhibitor caused fewer bleeding complications than a vitamin $\mathrm{K}$ antagonist plus a $\mathrm{P}_{2} \mathrm{Y}_{12}$ inhibitor plus aspirin. The rate of death from cardiovascular causes, myocardial infarction, or stroke was similar in the 3 treatment groups.

The RE-DUAL PCI trial (Randomized Evaluation of Dual Antithrombotic Therapy With Dabigatran Versus Triple Therapy With Warfarin in Patients With Nonvalvular Atrial Fibrillation Undergoing Percutaneous Coronary Intervention), ${ }^{4}$ in 2017, found that the combination of the DOAC dabigatran plus a $\mathrm{P}_{2} \mathrm{Y}_{12}$ inhibitor (clopidogrel or ticagrelor) caused fewer bleeding complications than warfarin plus a $\mathrm{P}_{2} \mathrm{Y}_{12}$ inhibitor plus aspirin, and was as effective.

The AUGUSTUS ${ }^{7}$ trial, in 2019, was a 2-by-2 factorial trial that assigned patients who were planning to take a $\mathrm{P}_{2} \mathrm{Y}_{12}$ inhibitor to receive apixaban or a vitamin $\mathrm{K}$ antagonist, and separately assigned them to receive aspirin or placebo. Apixaban, without aspirin, resulted in fewer bleeding events and hospitalizations without significant differences in the incidence of ischemic events.

The ENTRUST-AF PCI trial, ${ }^{8}$ also in 2019, further demonstrated that a DOAC (edoxaban) plus a P2Y $\mathrm{Y}_{12}$ inhibitor was noninferior to the combination of a vitamin $\mathrm{K}$ antagonist, $\mathrm{P}_{2} \mathrm{Y}_{12}$ inhibitor, and aspirin.

\section{PATIENTS AT HIGH RISK OF STROKE OR BLEEDING}

For patients at high risk of stroke and low risk of bleeding, it is not unreasonable to extend triple therapy for up to 1 month after PCI. ${ }^{1}$ However, after 1 month, double therapy should be used to reduce the risk of a bleeding event and death. ${ }^{1}$ Anticoagulation plus a single antiplatelet drug should be used for only up to 1 year before switching to single oral non-vitamin $\mathrm{K}$ antagonist therapy only. ${ }^{1}$

For patients at low stroke risk and high bleeding risk, anticoagulation plus singleantiplatelet therapy should be started immediately after PCI, but continued for only up to 6 months, followed by a non-vitamin $\mathrm{K}$ antagonist only. ${ }^{1}$ Furthermore, a proton-pump inhibitor can be considered, to further reduce the risk of bleeding in those at high risk during the period of anticoagulation and singleantiplatelet combination therapy.

\section{TOOLS TO HELP DECISION-MAKING}

The $\mathrm{CHA}_{2} \mathrm{DS}_{2}$-VASc score should be used to calculate the stroke risk in patients with atrial fibrillation. Points are given for congestive heart failure, hypertension, age greater than 65 (or 2 points for age > 75), diabetes, stroke ( 2 points), vascular disease, and female sex category, for a maximum of 9 points. A score of 2 or higher indicates a high risk of stroke $(\geq$ $2.2 \%$ per year). ${ }^{9}$

The HAS-BLED score can be used to estimate the risk of bleeding in a patient on anticoagulant therapy. ${ }^{6,9}$ Points are given for hypertension, abnormal renal function, abnormal liver function, stroke, bleeding, labile international normalized ratio, elderly status, use of drugs that predispose to bleeding, and use of alcohol. ${ }^{8}$ A score of 3 or more indicates a high risk of bleeding ( $\geq 5.8 \%$ per year).

\section{WHEN TO DISCONTINUE THERAPY}

Discontinuation of single-antiplatelet therapy after 1 year (or after 6 months in patients at high risk of bleeding but low risk of stroke) should follow the standard thromboembolism prevention protocol in patients with atrial fibrillation with an oral non-vitamin $\mathrm{K}$ antagonist only. ${ }^{9}$

The AFIRE trial (Atrial Fibrillation and Ischemic Events With Rivaroxaban), ${ }^{10}$ in 2019, demonstrated no decrease in therapeutic effects with rivaroxaban by itself compared 


\section{TABLE 1}

\section{Anticoagulation after percutaneous coronary intervention in patients with atrial fibrillation}

\begin{tabular}{|c|c|c|c|}
\hline Time after $\mathrm{PCl}$ & $\begin{array}{l}\text { Low risk of stroke, } \\
\text { low risk of bleeding }\end{array}$ & $\begin{array}{l}\text { High risk of stroke, } \\
\text { low risk of bleeding }\end{array}$ & $\begin{array}{l}\text { Low risk of stroke, } \\
\text { high risk of bleeding }\end{array}$ \\
\hline 0-1 month & DOAC plus $\mathrm{P} \mathrm{Y}_{12}$ inhibitor & $\begin{array}{l}\text { DOAC plus } \text { P2Y }_{12} \text { inhibitor } \\
\text { plus aspirin }\end{array}$ & DOAC plus $P_{2} Y_{12}$ inhibitor \\
\hline 1-6 months & DOAC plus $\mathrm{P}^{2} \mathrm{Y}_{12}$ inhibitor & DOAC plus $\mathrm{P}^{2} \mathrm{Y}_{12}$ inhibitor & DOAC plus $P 2 Y_{12}$ inhibitor \\
\hline 6-12 months & DOAC plus $\mathrm{P}_{2} \mathrm{Y}_{12}$ inhibitor & DOAC plus $\mathrm{P}_{2} \mathrm{Y}_{12}$ inhibitor & DOAC only \\
\hline $\begin{array}{l}\text { After } 12 \\
\text { months }\end{array}$ & $\begin{array}{l}\text { DOAC only, } \\
\text { discuss risks and benefits }\end{array}$ & $\begin{array}{l}\text { DOAC only, } \\
\text { discuss risks and benefits }\end{array}$ & $\begin{array}{l}\text { DOAC only, } \\
\text { discuss risks and benefits }\end{array}$ \\
\hline
\end{tabular}

with rivaroxaban combined with antiplatelet agents, and an increase in safety after 12 months.

For patients with $\mathrm{CHA}_{2} \mathrm{DS}_{2}$-VASc scores greater than 2, chronic anticoagulation should be advised. ${ }^{9}$ For those with a score of 1 or 2 , a conversation should be had regarding the risks and benefits. ${ }^{9}$ Those with a score of 0 should not continue anticoagulation, and can consider taking only a single antiplatelet agent. ${ }^{9}$

\section{TAKE-HOME MESSAGES}

- Triple therapy with anticoagulation plus 2 antiplatelet drugs is a very aggressive approach and carries a higher risk of bleeding

\section{REFERENCES}

1. Angiolillo DJ, Goodman SG, Bhatt DL, et al. Antithrombotic therapy in patients with atrial fibrillation treated with oral anticoagulation undergoing percutaneous coronary intervention: a North American perspective-2018 update. Circulation 2018; 138(5):527-536. doi:10.1161/CIRCULATIONAHA.118.034722

2. Dewilde WJ, Oirbans T, Verheugt FW, et al. Use of clopidogrel with or without aspirin in patients taking oral anticoagulant therapy and undergoing percutaneous coronary intervention: an open-label, randomised, controlled trial. Lancet 2013; 381(9872):1107-1115. doi:10.1016/S0140-6736(12)62177-1

3. Gibson CM, Mehran R, Bode C, et al. Prevention of bleeding in patients with atrial fibrillation undergoing PCI. N Engl J Med 2016; 375(25):2423-2434. doi:10.1056/NEJMoa1611594

4. Cannon CP, Bhatt DL, Oldgren J, et al. Dual antithrombotic therapy with dabigatran after PCl in atrial fibrillation. N Engl J Med 2017; 377(16):1513-1524. doi:10.1056/NEJMoa1708454

5. Bainey KR, Morais J, Zeymer U, Welsh RC. Atrial fibrillation with percutaneous coronary intervention: navigating the minefield of antithrombotic therapies. Atherosclerosis 2019; 289:118-125. doi:10.1016/j.atherosclerosis.2019.08.021

6. Chang TY, Lip GYH, Chen SA, Chao TF. Importance of risk reas- events and death than double therapy with anticoagulation plus a single antiplatelet drug, with no tradeoff in efficiency.

- In patients with high ischemic risk and low bleeding risk, anticoagulation plus 2 antiplatelet drugs can be given for up to 1 month after PCI before discontinuing 1 of the antiplatelet drugs, ie, aspirin. Table 1 summarizes the treatment protocol depending on a patient's ischemic and bleeding risk.

- The $\mathrm{CHA}_{2} \mathrm{DS}_{2}$-VASc and HAS-BLED scores should be used to determine a patient's thromboembolic risk and risk of a bleeding risk, respectively.

sessment in patients with atrial fibrillation in guidelines: assessing risk as a dynamic process. Can J Cardiol 2019; 35(5):611-618. doi:10.1016/j.cjca.2019.01.018

7. Lopes RD, Heizer G, Aronson R, et al. Antithrombotic therapy after acute coronary syndrome or $\mathrm{PCl}$ in atrial fibrillation. N Engl J Med 2019; 380(16):1509-1524. doi:10.1056/NEJMoa1817083

8. Vranckx P, Valgimigli M, Eckardt L, et al. Edoxaban-based versus vitamin $\mathrm{K}$ antagonist-based antithrombotic regimen after successful coronary stenting in patients with atrial fibrillation (ENTRUSTAF PCI): a randomised, open-label, phase $3 \mathrm{~b}$ trial. Lancet 2019; 394(10206):1335-1343. doi:10.1016/S0140-6736(19)31872-0

9. January CT, Wann LS, Alpert JS, et al. 2014 AHA/ACC/HRS guideline for the management of patients with atrial fibrillation: a report of the American College of Cardiology/American Heart Association Task Force on Practice Guidelines and the Heart Rhythm Society. J Am Coll Cardiol 2014; 64(21):e1-e76. doi:10.1016/j.jacc.2014.03.022

10. Yasuda S, Kaikita K, Akao M, et al. Antithrombotic therapy for atrial fibrillation with stable coronary disease. N Engl J Med 2019; 381(12):1103-1113. doi:10.1056/NEJMoa1904143

Address: Asim Kichloo, MD, Central Michigan University College of Medicine, 1000 Houghton Avenue, Saginaw, MI 48602;

kichlooasim@gmail.com 